\title{
COMPARISON OF RELATIVE PERTURBATIONS ON SPACECRAFT EARTH ORBITS
}

\author{
Nicholas Mastricola AND OSSama AbDelkhalik \\ Department of Mechanical Engineering - Engineering Mechanics, \\ Michigan Technical University, 815 R.L. Smith Bldg., \\ 1400 Townsend Dr., Houghton, MI 49931, USA. \\ npmastricola@gmail.com,ooabdelk@mtu.edu
}

\begin{abstract}
In this paper, the individual relativistic perturbations are simulated and compared, for two orbit cases: (1) a medium altitude eccentric earth orbit (MEO), and (2) a Molniya orbit. The simulations' results are presented in both graphical and statistical form. The simulation data reveals that the specific forces that arise from the relativistic perturbations, do manifest as variations in the classical orbital elements. The simulated data also show that the specific forces exhibit similar effects that materialize from commonly considered perturbations that are used in orbit design and maintenance.
\end{abstract}

ABSTRAK: Dalam kertaskerja ini, pengusikan relativistik disimulasi dan dibandingkan secara individu bagi dua kes orbit: (1) orbit eksentrik bumi ketinggian sederhana (MEO), dan (2) orbit Molniya. Hasil simulasi ini dibentangkan dalam bentuk grafik dan statistik. Data simulasi menunjukkan daya spesifik pengusikan relativistik timbul sebagai variasi bagi elemen orbit klasik. Data simulasi menunjukkan daya spesifik memberi kesan yang sama dengan pengusikan biasa yang digunakan dalam rekabentuk dan penyelenggaraan orbit.

KEYWORDS: relatistic perturbations; orbit perturbations

\section{INTRODUCTION}

In Newtonian mechanics, the natural motion of a spacecraft around a celestial body is described by a second order vectorial differential equation; assuming that the spacecraft is attracted only by the celestial body, which is assumed to be a perfect sphere [1]. This equation of motion is based on Newton's law of gravitation. Prior works in the literature have generalized the model of spacecraft motion to include the effect of gravity from a third body (other than the central body and the spacecraft) and continuous thrust forces [2]. The three-dimensional restricted four-body problem was also studied [3]. Lagrange points were also utilized to find low energy interplanetary trajectories [4]. All the work described above has been developed in a Newtonian framework; and exhaustive sets of numerical tools have been developed over years to carry out all orbital mechanics tasks including trajectory design, propagation, and determination. During the twentieth century, however, Einstein developed the special and general relativity theories. Einstein's field equations are the relativistic generalization of Newton's law of gravitation [5].

The General Theory of Relativity (GTR) has traditionally been looked at and utilized in the realms of theoretical and mathematical physics. The GRT was used to provide more accurate calculations for the purpose of matching observational data [6]. Several studies, however, have addressed the relativistic perturbations on low earth orbits. On one hand, some low earth missions require high precession navigation in which relativistic perturbations need to be accounted for. On the other hand, several measurements data 
were collected from low altitude satellites and hence models for the relativistic perturbations at low earth altitudes are used for verifications. Reference [7], for instance, presents numerical simulations for the overall general relativity theory perturbations, among several other perturbations, on low earth orbits. This paper, however, focuses on the quantification of the individual relativistic perturbations on earth orbits.

The Schwarzschild metric, $g_{\mu v}$, is implemented for space-time; and the line element in the space-time can be calculated by two simple contractions of the metric tensor with the differential length element (Schwarzchild solution [8]):

$$
d s^{s}=g_{\mu v} d x^{\mu} d x^{v}
$$

Where the differential length element $d x^{\mu}$ is represented by the space-time 4-vector as, $d x^{\mu}=\left[\begin{array}{llll}d t & d r & d \theta & d \phi\end{array}\right]^{T}$.

Where $r, \theta$, and $\phi$ are the polar coordinates, and $t$ is the time. The geodesic equations that determine the motion of a particle in a Schwarzchild-space are:

$$
\begin{aligned}
& 0=\frac{d}{d s}\left[\left(1-\frac{2 m}{r}\right) \frac{d t}{d s}\right] \\
& 0=\frac{d}{d s}\left[-\frac{r^{2}}{c^{2}} \frac{d \theta}{d s}\right]-\frac{1}{2}\left(-\frac{r^{2}}{c^{2}} 2 \sin \theta \cos \theta\right)\left(\frac{d \phi}{d s}\right)^{2} \\
& 0=\frac{d}{d s}\left[-\frac{r^{2}}{c^{2}} \sin ^{2} \theta \frac{d \phi}{d s}\right]
\end{aligned}
$$

Where $c$ is the speed of light, $m=\frac{G M}{c^{2}}$ is the Schwarzschild radius, $G$ is Newton's

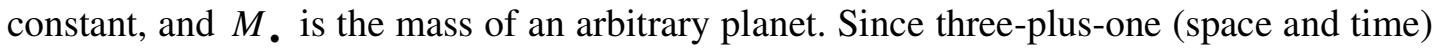
coordinates are now being used, an independent fourth equation is needed. For this, the first integral of Equations [5,9,10] is used [11]:

$$
1=\left(1-2 \frac{m}{r}\right)\left(\frac{d t}{d s}\right)^{2}-\frac{1}{c^{2}}\left(\left(1-2 \frac{m}{r}\right)^{-1}\left(\frac{d r}{d s}\right)^{2}+r^{2}\left(\frac{d \theta}{d s}\right)^{2}+r^{2} \sin ^{2}(\theta)\left(\frac{d \phi}{d s}\right)^{2}\right)
$$

It is possible to make the following two simplifying assumptions [10]: (1) the central body in a satellite-planet system is the only relativistically significant body, and (2) the influence of the relativistic forces is so slight that they may be thought of, and treated, as perturbations [10]. Applying these assumptions allows for the modeling of relativistic forces in the same manner that Newtonian gravitational forces and motion are modeled [12]:

$$
\ddot{\vec{r}}=-\frac{\mu}{r^{2}} \hat{r}+\vec{a}_{\text {pert }}
$$

The individual relativistic perturbing accelerations that make up $\vec{a}_{\text {pert }}$ are outlined section 2. Section 3 shows the numerical results for simulations of the individual relativistic perturbations on two orbits: a Medium Earth Orbit (MEO) and a Molniya orbit.

\section{ORBIT RELATIVISTIC PERTURBATIONS}

The special perturbation technique and Crowell's formulation were chosen to produce the numerical results shown in section 3 because of its rapid succession. The form of the relativistic perturbing terms used in the numerical simulations are based on those presented in [10]. This section introduces the acceleration terms that are used in the numerical simulations. 


\subsection{Central Body Terms}

The central body shape effects are modeled in the relativistic framework as a combination of two perturbation terms [13]: (1) the spherical perturbation term, and (2) the relativistic oblateness term. The spherical body perturbation term provides the acceleration of a test particle around a spherically symmetric central body $[8,14,13]$. The spherical body perturbation term can be modeled as:

$$
\vec{a}_{S T}=\frac{\mu_{\otimes}}{c^{2} r^{3}}\left\{\left[2(y+\beta) \frac{\mu_{\oplus}}{r}-\gamma(\dot{\vec{r}} \cdot \dot{\vec{r}})\right] \vec{r}+2(1+\gamma)(\vec{r} \cdot \dot{\vec{r}}) \dot{\vec{r}}\right\}
$$

where $\gamma$ and $\beta$ are the relativistic parameters, $r$ is the magnitude of the position vector $\vec{r}$, and $\mu_{\oplus}$ is the gravitational parameter of earth. The shape of earth is an oblate spheroid [12]. In non-relativistic theory, the earth's oblateness is modeled using derivatives of the zonal harmonic functions [12]. The most prominent of these is the second zonal harmonic term [9], which produces what is commonly refered to the $J_{2}$-term. The final perturbation acceleration for the $J_{2}$-term takes the form [12]:

$$
\vec{a}_{J 2}=-\frac{3 J_{2} \mu_{\oplus} R_{\oplus}^{2}}{2 r^{5}} x\left(1-\frac{5 z^{2}}{r^{2}}\right) y\left(1-\frac{5 z^{2}}{r^{2}}\right) z\left(3-\frac{5 z^{2}}{r^{2}}\right)
$$

where $(x, y, z)$ are the components of $\vec{r}, R_{\oplus}$ is the average earth radius, and $J_{2}$ is the earth's oblateness factor. This term does not take into account the relativistic effects acting on the system [14]. The relativistic oblateness term takes the form [14]:

$$
\begin{aligned}
\vec{a}_{R J 2}= & J_{2} \frac{\mu}{c^{2} r^{3}}\left(\frac{a_{\oplus}}{r}\right)^{2}\left\{2(\gamma+\beta) \frac{\mu_{\oplus}}{r}\left[\begin{array}{l}
x\left(2-9\left(\frac{z}{r}\right)^{2}\right) \\
y\left(2-9\left(\frac{z}{r}\right)^{2}\right) \\
z\left(5-9\left(\frac{z}{r}\right)^{2}\right)
\end{array}\right]-\frac{3}{2}\left(\frac{\dot{\vec{r}} \cdot \dot{\vec{r}}}{r}\right)\left[\begin{array}{l}
x\left(1-5\left(\frac{z}{r}\right)^{2}\right) \\
y\left(1-5\left(\frac{z}{r}\right)^{2}\right) \\
x\left(3-5\left(\frac{z}{r}\right)^{2}\right)
\end{array}\right]\right. \\
& \left.+3(1+\gamma)\left[\left(1-5\left(\frac{z}{r}\right)^{2}\right)(\vec{r} \cdot \dot{\vec{r}})+2 z \dot{z}\right] \dot{\vec{r}}\right\}
\end{aligned}
$$

where $a_{\oplus}$ is the earth semimajor axis. The relativistic oblateness term may be regarded as an updated version of the standard oblateness term, because the latter was the starting point to derive the necessary relativistic correction [14].

\subsection{Lense-Thirring and Rotational Energy Terms}

The Lense-Thirring perturbation term is referred to as a gravitomagnetic effect and arises from angular momentum of the central body [13]. This manifests itself by "dragging" a reference frame along an orbit in the direction of the the central body's rotation [12]. In other words, the Lense-Thirring effect forces the orbit to precess around the central body in the direction of the central body's rotation (frame dragging).

The Lense-Thirring perturbation is modeled as:

$$
\vec{a}_{L T}=L(1+\gamma) \frac{\mu_{\oplus}}{c^{2} r^{3}}\left[\frac{3}{r^{2}} \vec{r} \vec{J} *(\vec{r} \times \dot{\vec{r}})+\left(\dot{\vec{r}} \times \vec{J}^{*}\right)\right]
$$

where $\vec{J}^{*}$ is the the Specific polar moment of inertia $\left(1.3455 e 13 \mathrm{~m}^{2}\right)$, and $L$ is the Lense-Thirring parameter. Within the GTR framework, Lmay be taken to equal 1.0 [13]. Using this as a simplifying assumption, the classical form of the Lense-Thirring term takes the form [13]: 


$$
\begin{aligned}
& \vec{a}_{L T}=\frac{v}{r^{3}} \dot{y}+3 \frac{z}{r^{2}}(y \dot{z}-z \dot{y})-\dot{x}+3 \frac{z}{r^{2}}(z \dot{x}-x \dot{z}) 3 \frac{z}{r^{2}}(x \dot{y}-y \dot{x}) \\
& \text { where, } v=\frac{2}{5}(1-\gamma) \frac{\mu_{\oplus} R_{\oplus}^{2} \Omega_{\oplus}}{c^{2}}
\end{aligned}
$$

Equation (11) was used in the simulations presented in this paper.

The Lense-Thirring term does not give the entire picture of frame dragging because there is also the rotational energy perturbation acceleration, which is modeled as $[13,10]$ :

$$
\vec{a}_{R E}=\frac{3}{14} T_{\oplus}^{*}(1+\gamma) \frac{\mu_{\oplus}}{c^{2} r^{3}}\left(\frac{a_{\oplus}}{r}\right)^{2}\left\{\left(1-5\left(\frac{z}{r}\right)^{2}\right) \vec{r}+2\left(\vec{r} \cdot \hat{\Omega}_{\oplus}\right) \hat{\Omega}_{\oplus}\right\}
$$

Reference [13], however, has shown that the rotational energy term contributes small perturbing forces.

\subsection{Geodesic Precession Term}

The form of the geodesic precession acceleration that was used to create the data in this paper is [10]:

$$
\vec{a}_{G P}=-\frac{\mu_{\epsilon}}{c^{2} R_{\oplus \epsilon}^{3}}(1+2 \gamma)\left(\dot{\vec{R}}_{\oplus_{\epsilon}} \times \vec{R}_{{ }_{\oplus}}\right) \times \dot{\vec{r}}
$$

Where $\mu_{\epsilon}$ is the gravitational parameter of the sun, the relative position and velocity vectors of the earth with respect to the sun are $\vec{R}_{\oplus_{\epsilon}}$ and $\dot{\vec{R}}_{\oplus_{\epsilon}}$ respectively, and the velocity vector of the satellite with respect to the earth is $\vec{r}$.

\section{NUMERICAL RESULTS AND DISCUSSION}

All calculations for all terms were done in the earth-fixed coordinate frame. The earth's position and velocity data were generated using the HORIZONS system ${ }^{1}$, which is maintained by the Solar System Dynamics Group of the Jet Propulsion Laboratory (JPL) ${ }^{2}$.

Two orbits are considered for simulations: a Medium altitude Earth Orbit (MEO), and a Molniya orbit. The orbital elements for both orbits are listed in Table 1.

Table 1: Orbital Parameters for Test Orbit Cases.

\begin{tabular}{clll}
\hline Orbital Parameter & MEO & Molniya Orbit & Units \\
\hline$\theta_{\mathrm{o}}$ & 28.0000 & 28.0000 & degrees \\
$e_{\mathrm{o}}$ & 0.4500 & 0.7000 & NA \\
$a_{\mathrm{o}}$ & $1.34147272 \mathrm{E} 4$ & $2.66102228 \mathrm{E} 4$ & kilometers \\
$p_{\mathrm{o}}$ & $1.6982450 \mathrm{E} 4$ & $1.35712136 \mathrm{E} 4$ & kilometers \\
$i_{\mathrm{o}}$ & 32.0000 & 63.9000 & degrees \\
$\omega_{\mathrm{o}}$ & 18.0000 & 0.0000 & degrees \\
$\Omega_{\mathrm{o}}$ & 24.0000 & 0.0000 & degrees \\
Orbital Period $\Pi$ & 4.2952 & 12.0000 & hours \\
\hline
\end{tabular}

${ }^{1}$ http://ssd.jpl.nasa.gov/?horizons

${ }^{2}$ http://www.jpl.nasa.gov/ 
This section contains a discussion for the effects of the relativistic oblateness perturbation on two different orbit cases. The data presented includes orbital parameter differences plots as well as specific force magnitudes. This is followed by a comparison of the relativistic oblateness perturbation to the standard oblateness perturbation developed in the classical theory. A summery for the trends of all other relativistic perturbations is then presented. Detailed results for the other relativistic perturbations are not presented in this paper, but are available in [16].

\subsection{Relativistic Oblateness Orbital Element Data}

The effects of the relativistic oblateness perturbations on the MEO and the Molniya Orbit are discussed in this section. The difference plots were generated by taking the data from the desired perturbation and subtracting out the data that was produced by integrating the orbit without perturbation. It is possible to classify the modulations experienced by the orbital elements into three classes [9]. They are: (1) short term periodic, (2) long term periodic, and (3) secular (adapted from [9]). A secular variation is a modulation that has a consistent trend over a long period of time.

\subsubsection{The MEO}

The true anomaly $\theta$ and the magnitude of spacecraft's position vector $r$ exhibit periodicity, as seen in Fig. 1 and Fig. 2 respectively. Both are short term periodic. The periodicity of these difference plots suggests that these elements experience phasing. Both orbits, while exhibiting a strong periodic behavior, also show secular characteristics as well. As seen in Fig. 1 that the true anomaly difference increases with time. However the difference plot of the spacecraft position vector's magnitude, seen in Fig. 2, shows a full reversal. This full reversal implies that this difference quantity has a lead-lag relationship with the unperturbed orbit.

The remainder of the orbital elements displayed in Fig. 1 and Fig. 2 exhibit predominately secular behavior. On a short time scale, all of these elements do show some short term periodicity. However, the short term periodicity is negligible in effect compared to the secular modulation.

\subsubsection{Molniya Orbit Case}

The true anomaly $\theta$, the angle of inclination $i$, and the magnitude of the spacecraft position vector $r$ exhibit periodic behavior. The inclination is the only true periodic element, which exhibits both short and long term periodicity. The difference plot of the 

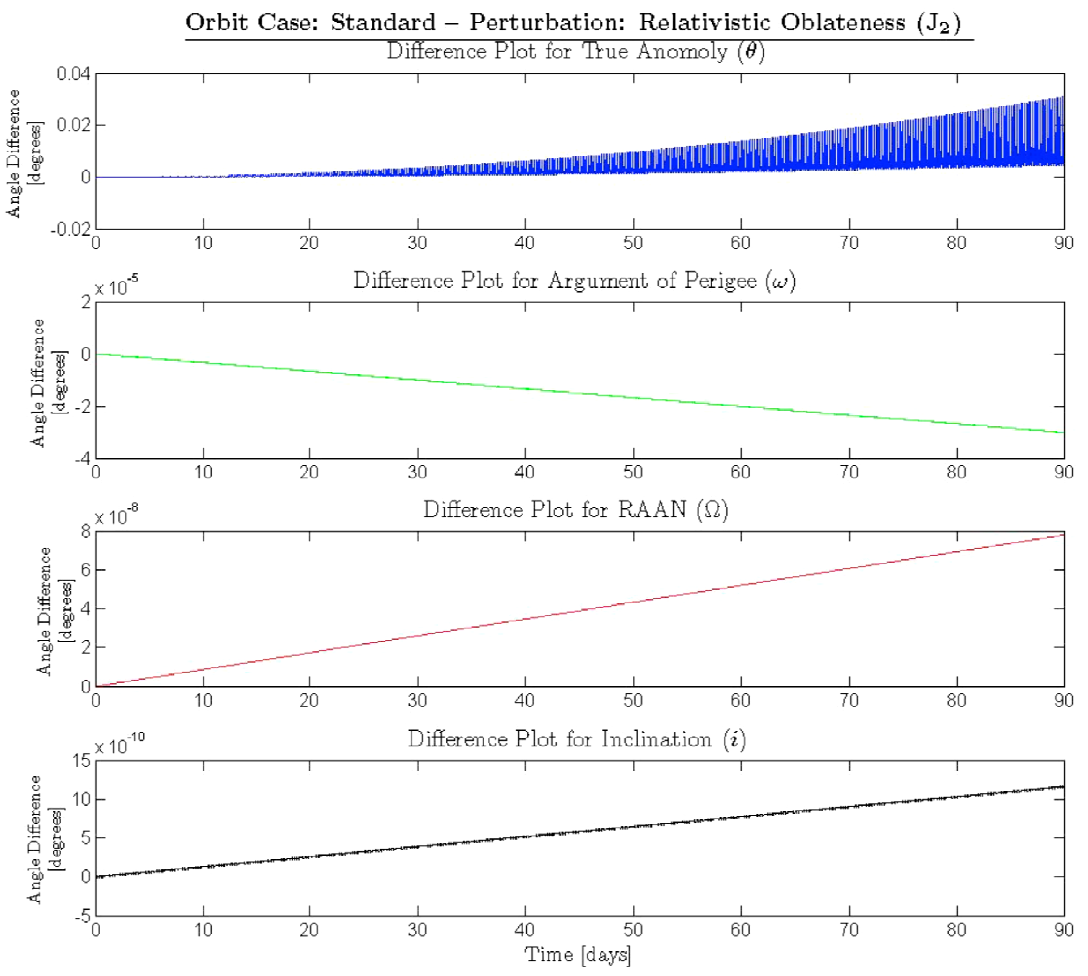

Fig. 1: MEO Angular Differences for Relativistic Oblateness versus Time.
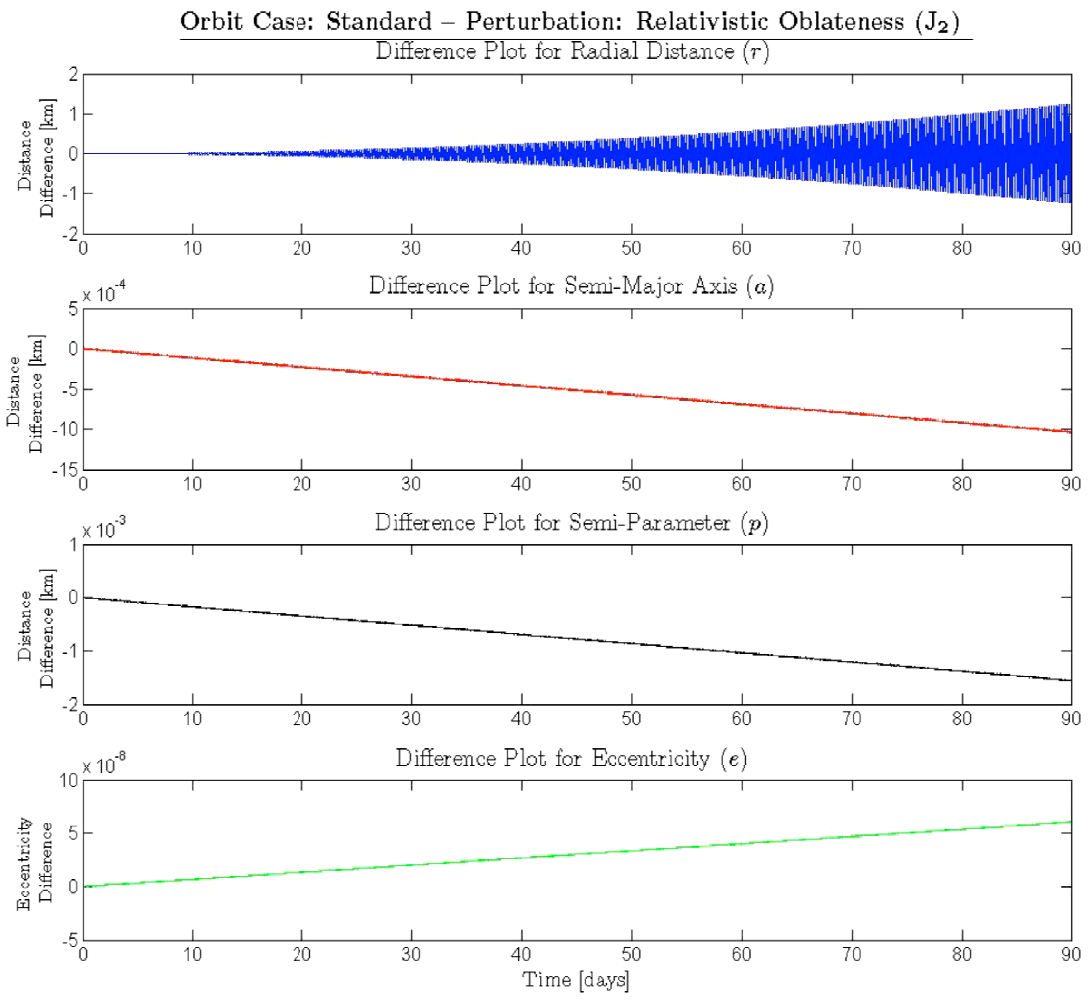

Fig. 2: MEO Distance Differences for Relativistic Oblateness versus Time. 
true anomaly $\theta$, shown in Fig. 3, presents with both short term periodic trends and a secular component. The difference plot of $r$, seen in Fig. 4, does show phasing behavior. This phasing is evident because the difference between the perturbed and unperturbed orbits does go to zero. Even though this plot exhibits increasing phase, it is not a true secular variation. It can be seen from Figure 4 that the semi-major axis $a$, the semiparameter $p$, and the eccentricity $e$ experience secular behavior and are all decreasing further from their values in the unperturbed orbit.
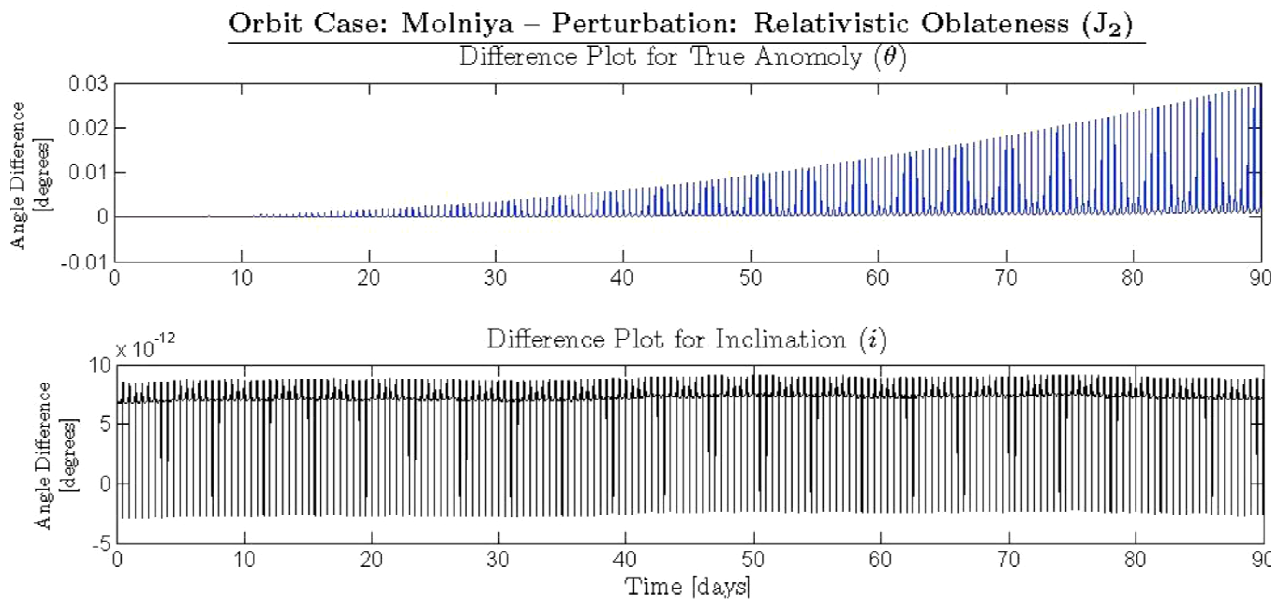

Fig. 3: Molniya Orbit Angular Differences for Relativistic Oblateness versus Time.
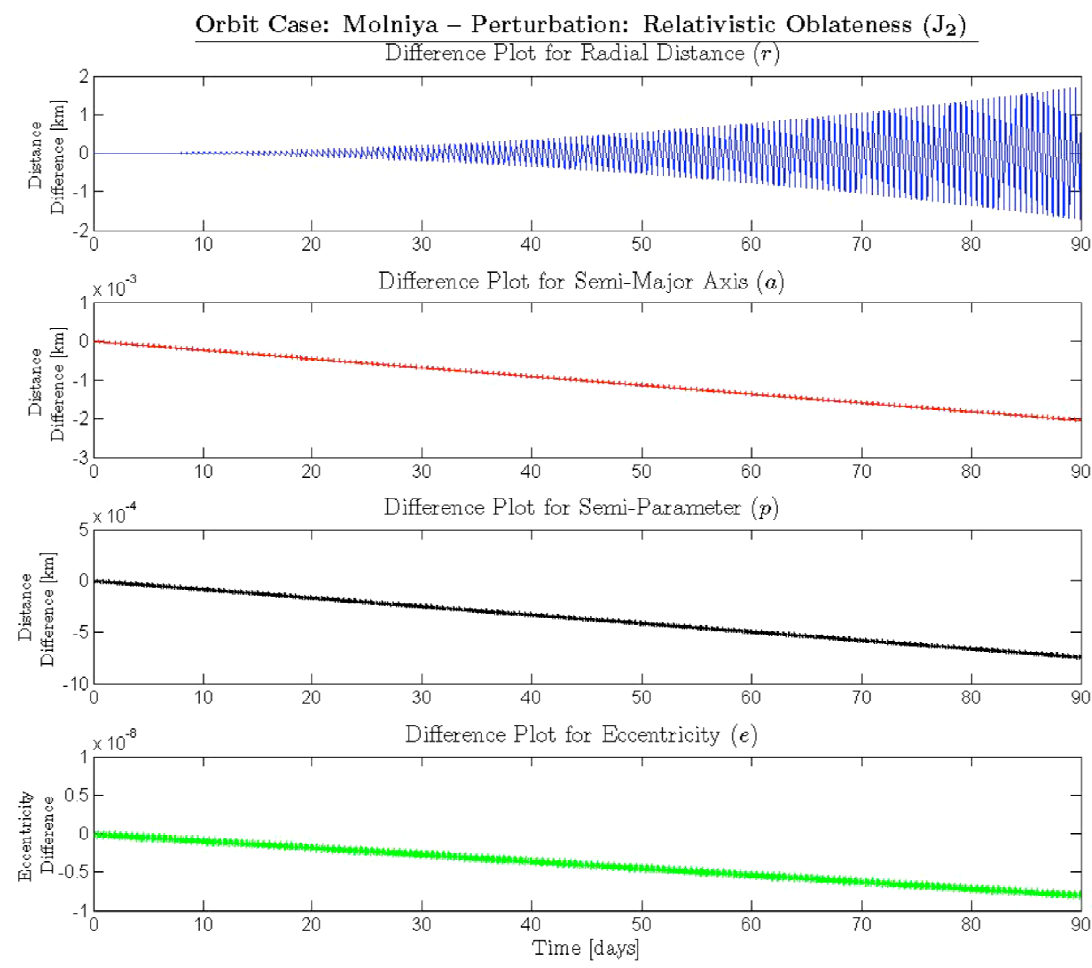

Fig. 4: Molniya Orbit Distance Differences for Relativistic Oblateness versus Time. 


\subsection{Discussion of Orbital Elements Data}

Comparisons between the relativistic oblateness and the standard oblateness perturbations are summarized in Tables 2 and 3. Table 2 displays the type of modulation (and trend where applicable) exhibited by each of the orbital elements. The minimum, maximum, mean and median values of the data contained in Table 2 are shown in Table 3.

Table 2: Summary of Data Trends.

\begin{tabular}{|c|c|c|c|c|c|}
\hline & \multicolumn{2}{|c|}{ Standard Oblateness } & \multicolumn{2}{|c|}{ Relativistic Oblateness } \\
\hline & & MEO & Molniya Orbit & MEO & Molniya Orbit \\
\hline$\theta$ & $\begin{array}{l}\text { Variation } \\
\text { Trend }\end{array}$ & $\begin{array}{l}\text { Sec. and Per. } \\
\text { Decreasing }\end{array}$ & $\begin{array}{l}\text { Sec. and Per. } \\
\text { Decreasing }\end{array}$ & $\begin{array}{l}\text { Sec. with Per. } \\
\text { Increasing }\end{array}$ & $\begin{array}{l}\text { Sec. with Per. } \\
\text { Increasing }\end{array}$ \\
\hline$a$ & $\begin{array}{l}\text { Periodic } \\
\text { na }\end{array}$ & $\begin{array}{c}\text { Periodic } \\
\text { na }\end{array}$ & $\begin{array}{c}\text { Secular } \\
\text { Decreasing }\end{array}$ & $\begin{array}{c}\text { Secular } \\
\text { Decreasing }\end{array}$ & $\begin{array}{c}\text { Periodic } \\
\text { na }\end{array}$ \\
\hline$e$ & Periodic & $\begin{array}{l}\text { Periodic } \\
\text { na }\end{array}$ & $\begin{array}{l}\text { Secular } \\
\text { na }\end{array}$ & $\begin{array}{c}\text { Secular } \\
\text { Increasing }\end{array}$ & $\begin{array}{c}\text { Periodic } \\
\text { Decreasing }\end{array}$ \\
\hline$p$ & $\begin{array}{l}\text { Variation } \\
\text { Trend }\end{array}$ & $\begin{array}{c}\text { Periodic } \\
\text { na }\end{array}$ & $\begin{array}{c}\text { Periodic } \\
\text { na }\end{array}$ & $\begin{array}{c}\text { Secular } \\
\text { Decreasing }\end{array}$ & $\begin{array}{c}\text { Secular } \\
\text { Decreasing }\end{array}$ \\
\hline$\omega$ & $\begin{array}{l}\text { Variation } \\
\text { Trend }\end{array}$ & $\begin{array}{c}\text { Secular } \\
\text { Decreasing }\end{array}$ & $\begin{array}{l}\text { na } \\
\text { na }\end{array}$ & $\begin{array}{c}\text { Secular } \\
\text { Decreasing }\end{array}$ & $\begin{array}{l}\text { na } \\
\text { na }\end{array}$ \\
\hline$i$ & $\begin{array}{l}\text { Variation } \\
\text { Trend }\end{array}$ & $\begin{array}{c}\text { Periodic } \\
\text { na }\end{array}$ & $\begin{array}{c}\text { Periodic } \\
\text { na }\end{array}$ & $\begin{array}{l}\text { Secular } \\
\text { Increasing }\end{array}$ & $\begin{array}{c}\text { Periodic } \\
\text { na }\end{array}$ \\
\hline
\end{tabular}

Table 3: Summary of Data Values.

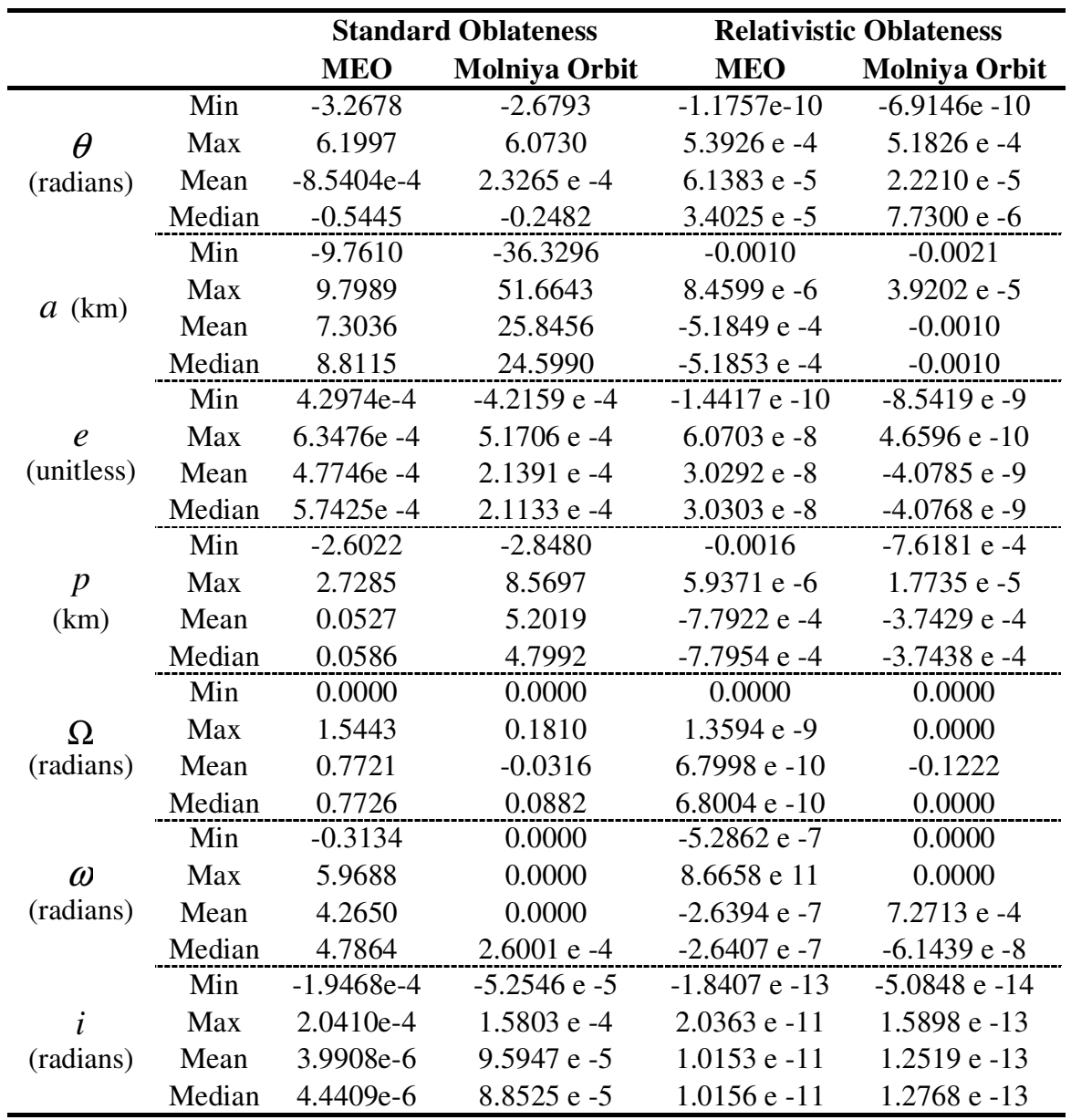




\subsubsection{Data Trends Analysis}

There are similarities between the standard and relativistic oblateness perturbations trends. Examples of these similarities are the true anomaly $\theta$ and the argument of periapsis $\omega$. The determination of $\omega$ have been labeled as "na" because the inclination of the Molniya orbit is such that the rate of change of $\omega$ vanishes. It is possible to see that the previously mentioned elements have the same type of modulation in each orbit case for their respective perturbations. There is a change in the trends exhibited by the true anomaly. The standard oblateness perturbation forces a decrease in this quantity. Whereas the relativistic oblateness perturbation induces an increase.

The same pattern is seen in the semi-major axis $a$ and semi-parameter $p$. Both of these elements have been shown to only exhibit purely periodic modulation under the standard oblateness perturbation [9]. When the relativistic oblateness perturbation is applied it is seen that both of these elements modulate in a secular fashion with a decreasing trend.

Eccentricity's, $e$, modulation type switches to secular with the implementation of the relativistic perturbation in the same manner as the modulation type of $a$ and $p$ in the preceding paragraph. However, there is a unique discrepancy of how the relativistic oblateness perturbation manifest in the MEO compared to how the perturbation manifests in the Molniya orbit. The relativistic oblateness perturbation effects the test orbits' eccentricity in the opposite manner -- i.e. the MEO's eccentricity increases while the Molniya orbit's decreases. In both trial orbits, the inclination $i$ experienced periodic modulation when the standard oblateness is simulated. This is in agreement with the proof provided in [9]. This is the same type of variation that is exhibited with the Molniya orbit when the relativistic oblateness perturbation is implemented. However, the MEO's inclination, under the relativistic oblateness perturbation, exhibits an increasing secular variation.

\subsubsection{Discussion of Data Values}

Table 3 shows the minimums, maximums, means, and medians of the difference data for the orbital elements. The true anomaly, $\theta$, is more effected for the MEO in both the standard and relativistic oblateness. However, the difference values are extremely close between each orbit case. This implies that there is very little difference between the different perturbation on this orbital parameter. The orders of magnitude for all of the comparison values (minimum, maximum, mean, and median) differ between half to one order of magnitude.

The semi-major axis is more effected for the Molniya orbit case under both perturbations. For the standard oblateness, the semi-major axis difference values are approximately one order of magnitude higher. This equates to a maximum instantaneous difference of approximately 51 kilometers. However, these difference values do not contribute to a secular change in this parameter as was mentioned previously. The difference values for the relativistic perturbation do contribute to the negative secular deviations pointed out in the last section.

The MEO's eccentricity, $e$, is more effected than the Molniya's eccentricity in both the standard and relativistic oblateness perturbations. The order of magnitudes of the comparison values are all similar in the case of the standard perturbation. However, in the case with the relativistic perturbation, there are order of magnitude differences. These 
differences are seen in all of the comparison values. In general, the order of magnitude differences are between one and two orders of magnitude lower for the Molniya orbit.

The semi-parameter, $p$, is more effected for the Molniya orbit under the standard oblateness perturbation; however, the MEO's semi-parameter is more effected under the influence of the relativistic oblateness perturbation. There is a difference of two orders of magnitude difference higher in the mean and median comparison values under the standard perturbation. In comparison, the MEO case, while under the influence of the relativistic perturbation, is approximately one order of magnitude higher. However, the order of magnitude analysis for the the orbit cases under the relativistic perturbations is inconclusive. Therefore, the same argument could be made for the Molniya orbit case.

For the inclination, $i$, the Molniya orbit is more effected under the standard perturbation. The MEO's inclination is more effected under the relativistic perturbation. Under the standard perturbation, there is a one order of magnitude difference in all comparison values. Under the relativistic oblateness perturbation, there is a one order of magnitude difference in the minimum comparison value and a two order of magnitude difference in all other comparison values.

\subsection{Discussion of Specific Force Data}

The specific force components $(\hat{I}, \hat{J}, \hat{K})$, as well as the magnitude, that manifests from the relativistic oblateness perturbation is shown in Fig. 5. These quantities were calculated through a post processing routine that used the outputted state vectors.

\subsubsection{Relativistic Oblateness Perturbation}

For both the MEO and the Molyina cases, all of the components and the magnitude of the specific force plots shown in Fig. 5 and Fig. 6 exhibit short term periodicity. All of the components also show full reversal. The mean value of the components, as well as the magnitude, also remains relatively constant.
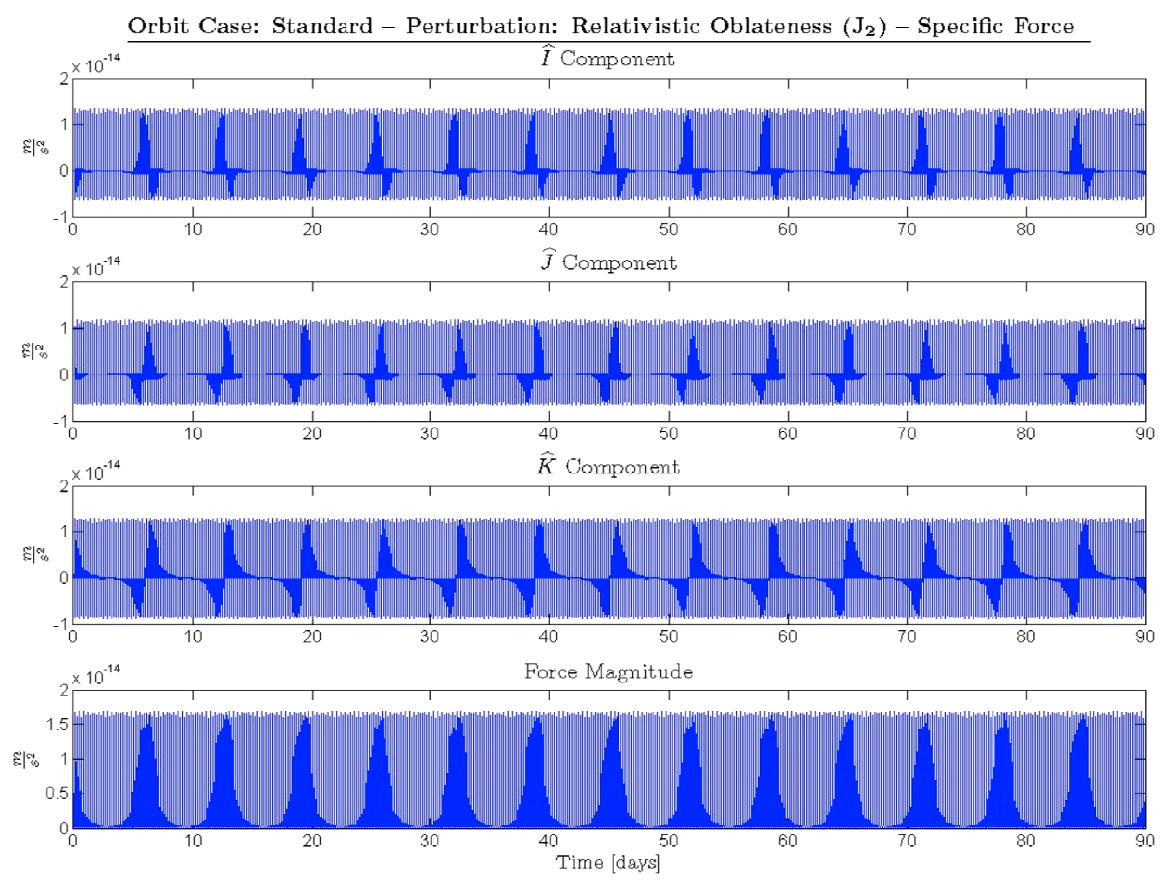

Fig. 5: MEO Specific Force for Relativistic Oblateness versus Time. 

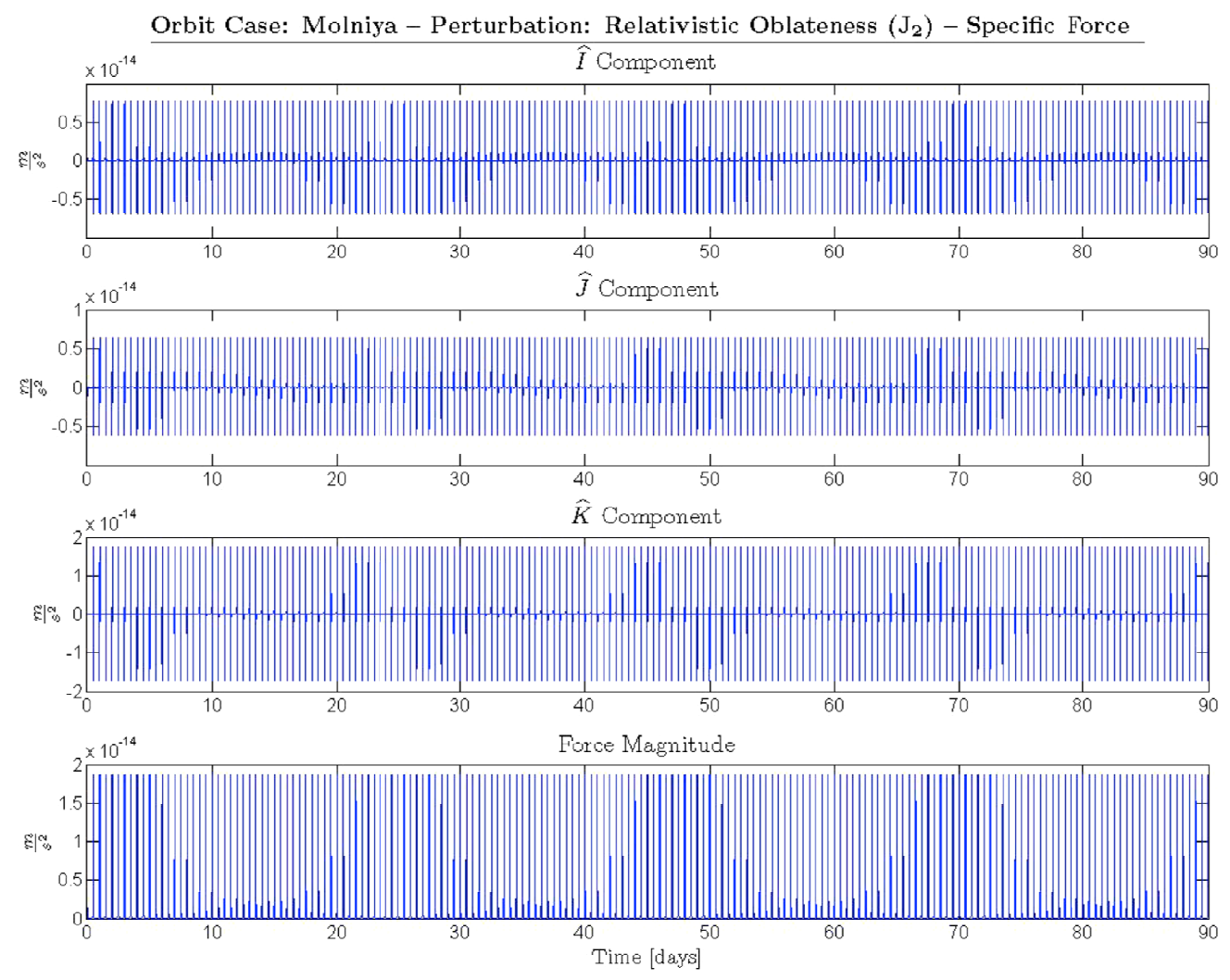

Fig. 6: Molniya Orbit Specific Force for Relativistic Oblateness versus Time.

\subsubsection{Comparison of Specific Force Data}

Table 4 shows the specific forces that are generated under the standard and relativistic perturbations for the two different test case orbits. The component values shown in Table 4 are expressed in the geocentric coordinates.

Table 4: Summary of Specific Force Data Values in Newtons.

\begin{tabular}{|c|c|c|c|c|c|}
\hline & & \multicolumn{2}{|c|}{ Standard Oblateness } & \multicolumn{2}{|c|}{ Relativistic Oblateness } \\
\hline & & MEO & $\begin{array}{l}\text { Molniya } \\
\text { Orbit }\end{array}$ & MEO & $\begin{array}{l}\text { Molniya } \\
\text { Orbit }\end{array}$ \\
\hline \multirow{4}{*}{$\begin{array}{c}x \\
\text { Component }\end{array}$} & Min & -3.0423 e -6 & -3.2203 e -6 & $-6.3920 \mathrm{e}-15$ & -6.7929 e -15 \\
\hline & Max & 7.6572 e -6 & 6.4808 e -6 & $1.3470 \mathrm{e}-14$ & $7.7277 \mathrm{e}-15$ \\
\hline & Mean & $1.2876 \mathrm{e}-7$ & $-3.9026 \mathrm{e}-11$ & $2.9774 \mathrm{e}-16$ & $-2.2701 \mathrm{e}-17$ \\
\hline & Median & -3.2454 e -8 & -5.0618 e -9 & $-6.9152 \mathrm{e}-17$ & $-1.5839 \mathrm{e}-18$ \\
\hline \multirow{4}{*}{$\begin{array}{c}y \\
\text { Component }\end{array}$} & Min & -2.2845 e -6 & -2.4222 e -6 & $-6.5751 \mathrm{e}-15$ & -6.1795 e -15 \\
\hline & Max & 6.2540 e -6 & $2.0221 \mathrm{e}-6$ & $1.1796 \mathrm{e}-14$ & $6.3781 \mathrm{e}-15$ \\
\hline & Mean & $1.5400 \mathrm{e}-7$ & -4.3278 e -10 & 9.3342 e -17 & $2.4601 \mathrm{e}-19$ \\
\hline & Median & $-1.8304 \mathrm{e}-8$ & -4.4576 e -10 & -6.4501 e -17 & $2.8740 \mathrm{e}-21$ \\
\hline \multirow{4}{*}{$\begin{array}{c}z \\
\text { Component }\end{array}$} & Min & $-7.9387 \mathrm{e}-6$ & $-4.7849 \mathrm{e}-6$ & $-8.7283 \mathrm{e}-15$ & $-1.7159 \mathrm{e}-14$ \\
\hline & Max & $6.6685 \mathrm{e}-6$ & 4.7861 e -6 & $1.2881 \mathrm{e}-14$ & $1.7461 \mathrm{e}-14$ \\
\hline & Mean & -3.9613 e -7 & $1.5461 \mathrm{e}-10$ & $2.5341 \mathrm{e}-16$ & 6.7349 e -19 \\
\hline & Median & $1.6383 \mathrm{e}-7$ & 6.2050 e -12 & $-4.7312 \mathrm{e}-17$ & $1.8596 \mathrm{e}-20$ \\
\hline \multirow{4}{*}{ Magnitude } & Min & $1.6380 \mathrm{e}-7$ & $6.2615 \mathrm{e}-9$ & $1.0676 \mathrm{e}-16$ & $1.6441 \mathrm{e}-18$ \\
\hline & $\operatorname{Max}$ & 8.8841 e -6 & 6.4826 e -6 & $1.6818 \mathrm{e}-14$ & $1.8775 \mathrm{e}-14$ \\
\hline & Mean & 1.4639 e -6 & $3.9380 \mathrm{e}-7$ & $2.2605 \mathrm{e}-15$ & $8.5347 \mathrm{e}-16$ \\
\hline & Median & $3.8642 \mathrm{e}-7$ & $1.2752 \mathrm{e}-8$ & $2.6582 \mathrm{e}-16$ & $3.6663 \mathrm{e}-18$ \\
\hline
\end{tabular}


Under the influence of the standard oblateness perturbation the Molniya orbit is less effected. The maximum order of magnitude differences in the specific force components is two. However, this trend is also echoed in the magnitude values of specific force.

The MEO is also more effected when subjected to the relativistic oblateness perturbation. Three is the maximum order of magnitude between specific force values while the different orbit cases are under the influence of the relativistic perturbation. The same conclusion may also be drawn from the magnitude data comparison values.

Using the data contained int Table 4 it is also possible to draw the conclusion that the relativistic oblateness perturbations are considerably weaker then the standard oblateness perturbation. As can be seen in Table 4, there are up to a thirteen orders of magnitude difference between the specific forces generated by the standard and relativistic perturbation.

\subsection{Other Relativistic Perturbations}

The output data from simulations for all relativistic perturbations described in section 2 is huge. For a concise presentation of the results, only the trends of the relativistic perturbations on the MEO orbit are listed in Table 5. The minimum, maximum, mean, and median values of the specific force magnitudes, on the MEO, are listed in Table 6. Detailed results are available in [16].

Table 5: Summarized Orbital Parameter Data for Standard Orbit Petrubations.

\begin{tabular}{|c|c|c|c|c|c|c|}
\hline & & Spherical & $\begin{array}{c}\text { Lense- } \\
\text { Thirring }\end{array}$ & $\begin{array}{l}\text { Relativistic } \\
\text { Oblateness }\end{array}$ & $\begin{array}{c}\text { Rotational } \\
\text { Energy }\end{array}$ & $\begin{array}{c}\text { Geodesic } \\
\text { Precession }\end{array}$ \\
\hline$\theta$ & $\begin{array}{l}\text { Variation } \\
\text { Trend }\end{array}$ & $\begin{array}{l}\text { Sec. with Per. } \\
\text { Increasing }\end{array}$ & $\begin{array}{l}\text { Sec. with Per. } \\
\text { Increasing }\end{array}$ & $\begin{array}{l}\text { Sec. with Per. } \\
\text { Increasing }\end{array}$ & $\begin{array}{l}\text { Sec. with Per. } \\
\text { Increasing }\end{array}$ & $\begin{array}{l}\text { Sec. with Per. } \\
\text { Increasing }\end{array}$ \\
\hline$a$ & $\begin{array}{l}\text { Variation } \\
\text { Trend }\end{array}$ & $\begin{array}{l}\text { Sec. with Per. } \\
\text { Decreasing }\end{array}$ & $\begin{array}{c}\text { Secular } \\
\text { Decreasing }\end{array}$ & $\begin{array}{c}\text { Secular } \\
\text { Decreasing }\end{array}$ & $\begin{array}{c}\text { Secular } \\
\text { Decreasing }\end{array}$ & $\begin{array}{c}\text { Secular } \\
\text { Decreasing }\end{array}$ \\
\hline$e$ & $\begin{array}{l}\text { Variation } \\
\text { Trend }\end{array}$ & $\begin{array}{c}\text { Secular } \\
\text { Increasing }\end{array}$ & $\begin{array}{l}\text { Sec. with Per. } \\
\text { Increasing }\end{array}$ & $\begin{array}{l}\text { Secular } \\
\text { Increasing }\end{array}$ & $\begin{array}{l}\text { Secular } \\
\text { Increasing }\end{array}$ & $\begin{array}{l}\text { Secular } \\
\text { Increasing }\end{array}$ \\
\hline$p$ & $\begin{array}{l}\text { Variation } \\
\text { Trend }\end{array}$ & $\begin{array}{c}\text { Secular } \\
\text { Decreasing }\end{array}$ & $\begin{array}{c}\text { Secular } \\
\text { Decreasing }\end{array}$ & $\begin{array}{l}\text { Secular } \\
\text { Decreasing }\end{array}$ & $\begin{array}{c}\text { Secular } \\
\text { Decreasing }\end{array}$ & $\begin{array}{c}\text { Secular } \\
\text { Decreasing }\end{array}$ \\
\hline$\Omega$ & $\begin{array}{l}\text { Variation } \\
\text { Trend }\end{array}$ & $\begin{array}{l}\text { Indeterminate } \\
\text { Indeterminate }\end{array}$ & $\begin{array}{l}\text { Secular } \\
\text { Increasing }\end{array}$ & $\begin{array}{l}\text { Secular } \\
\text { Increasing }\end{array}$ & $\begin{array}{c}\text { Secular } \\
\text { Decreasing }\end{array}$ & $\begin{array}{l}\text { Secular } \\
\text { Increasing }\end{array}$ \\
\hline$\omega$ & $\begin{array}{l}\text { Variation } \\
\text { Trend }\end{array}$ & $\begin{array}{c}\text { Secular } \\
\text { Increasing }\end{array}$ & $\begin{array}{c}\text { Secular } \\
\text { Decreasing }\end{array}$ & $\begin{array}{c}\text { Secular } \\
\text { Decreasing }\end{array}$ & $\begin{array}{c}\text { Secular } \\
\text { Decreasing }\end{array}$ & $\begin{array}{c}\text { Secular } \\
\text { Decreasing }\end{array}$ \\
\hline$i$ & $\begin{array}{l}\text { Variation } \\
\text { Trend }\end{array}$ & $\begin{array}{l}\text { Indeterminate } \\
\text { Indeterminate }\end{array}$ & $\begin{array}{c}\text { Periodic } \\
\text { na }\end{array}$ & $\begin{array}{l}\text { Secular } \\
\text { Increasing }\end{array}$ & $\begin{array}{c}\text { Secular } \\
\text { Decreasing }\end{array}$ & $\begin{array}{c}\text { Secular } \\
\text { Decreasing }\end{array}$ \\
\hline
\end{tabular}

Table 6: Specific Force Magnitude Data Summary for Standard Case in $\mathrm{m} / \mathrm{s}^{2}$

\begin{tabular}{clllll}
\hline & Spherical & $\begin{array}{l}\text { Lense- } \\
\text { Thirring }\end{array}$ & $\begin{array}{l}\text { Relativistic } \\
\text { Oblateness }\end{array}$ & $\begin{array}{l}\text { Rotational } \\
\text { Energy }\end{array}$ & $\begin{array}{l}\text { Geodesic } \\
\text { Precession }\end{array}$ \\
\hline Min & $3.4830 \mathrm{e}-7$ & $4.9138 \mathrm{e}-11$ & $1.0676 \mathrm{e}-16$ & $2.0045 \mathrm{e}-18$ & $1.4906 \mathrm{e}-14$ \\
Max & $5.2509 \mathrm{e}-12$ & $2.6990 \mathrm{e}-9$ & $1.6818 \mathrm{e}-14$ & $6.7816 \mathrm{e}-16$ & $4.2298 \mathrm{e}-14$ \\
Mean & $1.745 \mathrm{e}-12$ & $5.3501 \mathrm{e}-10$ & $2.2605 \mathrm{e}-15$ & $8.0623 \mathrm{e}-15$ & $2.6379 \mathrm{e}-14$ \\
Median & $9.5935 \mathrm{e}-13$ & $1.3613 \mathrm{e}-10$ & $2.6582 \mathrm{e}-16$ & $6.5495 \mathrm{e}-18$ & $2.4894 \mathrm{e}-14$ \\
\hline
\end{tabular}

\section{CONCLUSION}

The model that was derived and utilized in this research is essentially a modified form of the model that is used in classical Newtonian orbit theory. The relativistic perturbation terms that were used in the models, while being derived in a fully relativistic format, were implemented in the same manner as a standard perturbing term for the methods. This 
implementation was possible because of the form of the relativistic perturbations could be manipulated into a form that allowed them to be treated as a standard perturbation term.

The difference in orbital parameter deviation, specific force components, and specific force magnitudes were easily seen in the oblateness perturbations presented in the last section because the same effect was looked at, and derived, in both non-relativistic and relativistic frameworks. The differences that were exhibited between the effects produced by the non-relativistic perturbing terms and the relativistic perturbing terms are significant, albeit the the relativistic effects are comparatively smaller then the non-relativistic terms.

\section{REFERENCES}

[1] R. H. Battin. Battin, Richard H. "An Introduction to the Mathematics and Methods of Astrodynamics". AIAA, 1987., AIAA Education Series, New York, 1987."

[2] BL Pierson and CA Kluever. Pierson, Bion L., and Craig A. Kluever. "Three-stage approach to optimal low-thrust earth-moon trajectories". Journal of the Astronautical Sciences, 42(3):269-283, 1994. 42.3 (1994): 269-283.

[3] Nima Assadian, Seid H. Pourtakdoust. "Multiobjective genetic optimization of Earth-Moon trajectories in the restricted four-body problem". Advances in Space Research, 45(3):398409, 2010. 45.3 (2010): 398-409.

[4] Lo, M. "Invariant Manifolds, the Spatial Three-Body Problem and Space Mission Design." Astrodynamics Specialist Meeting”. Quebec City, Canada, (AAS 01-31), August 2001. . American Astronautical Society, 2001.

[5] Ji ṛ Bi çák. Bičák, Jiří. "Selected Solutions of Einstein's Field Equations: Their Role in General Relativity and Astrophysics." Einstein's Field Equations and Their Physical Implications. pages 1-126. Springer Berlin Heidelberg, Berlin/Heidelberg, 2000. 1-126.

[6] Thibault Damour and Michael Soffel and Chongming Xu. "General-Relativistic Celestial Mechanics. IV. Theory of Satellite Motion.” The American Physical Society, 49(2), Jan. 15, 1994. 49.2 (1994): 618.

[7] Eshangh ,Mehdi, and Mehdi. Najafi Alamdari. "Perturbations in orbital elements of a low earth orbiting satellite". Journal of the Earth \& space Physics, 33(1):1-12, 2007. 33.1 (2007): 1-12.

[8] Ray D'Inverno and Alex Harvey. "Introducing Einstein's Relativity". Oxford: Oxford University Press, 1992. Great Clarendon Street, Oxford OX2 6DP,

[9] Leon Blitzer. "Astronautics 453- Handbook of Orbital Perturbations". University of Arizona (1970).

[10] Chia-Chun George Chao. "Applied Orbit Perturbation and Maintenance". The Aerospace Press, El Segundo, California, 2005.

[11] Pamela A. Geisler. "Planetary Orbits in General Relativity". The Astronomical Journal, 68(9):715-717, 1963. 68.9 (1963): 715-17.

[12] David A. Vallado. "Fundamentals of Astrodynamics and Applications of Space Technology Library". Microcosm Press and Springer, 2007.

[13] Glenn Earl, Peterson. "Estimation of the Lense - Thirring Precession Using Laser - Ranged Satellites"., The University of Texas at Austin, 1997.

[14] Michael H. Soffel. "Relativity in Astrometry". Celestial Mechanics and Geodesy of Astronomy and Astrophysics." Springer - Verlag, Berlin, 1989.

[15] $\varnothing$ yvind Grø $\mathrm{n}$ and Sigbjø rn Hervik. Grøn, Øyvind, and Sigbjorn Hervik "Einstein's General Theory of Relativity with Modern Applications in Cosmology". Springer Science, 2007. + Business Media, LLC, 223 Spring Street, New York, NY 10013, USA, 2007. 
[16] Nicholas Mastricola. "Quantification of Relativistic Perturbation Forces on spacecraft Trajectories." Master's thesis, Michigan Technological University, 1400 Townsend Dr., Houghton, MI 49931, 2010. 